\title{
Transmission Dynamics of an Outbreak of the COVID-19 Delta Variant B.1.617.2 — Guangdong Province, China, May-June 2021
}

\author{
Meng Zhang ${ }^{1, \alpha}$; Jianpeng Xiao ${ }^{2, \alpha}$; Aiping Deng'; Yingtao Zhang'; Yali Zhuang'; Ting $\mathrm{Hu}^{1}$; Jiansen $\mathrm{Li}^{1}$; \\ Hongwei Tu' ${ }^{1}$; Bosheng $\mathrm{Li}^{1}$; Yan $\mathrm{Zhou}^{2,3}$; Jun Yuan'; Lei Luo'; Zimian Liang'; Youzhi Huang; \\ Guoqiang $\mathrm{Ye}^{7}$; Mingwei $\mathrm{Ca}^{8}$; Gongli Li'; Bo Yang ${ }^{10}$; Bin $\mathrm{Xu}^{11}$; Ximing Huang ${ }^{12}$; \\ Yazun Cui ${ }^{13}$; Dongsheng Ren ${ }^{14}$; Yanping Zhang ${ }^{15}$; Min Kang ${ }^{1,3, *}$; Yan Li $^{1, * t}$
}

On May 21, 2021, a local case of coronavirus disease 2019 (COVID-19) was confirmed in a 75-year-old woman (experienced onset of symptoms on May 18) in Liwan District, Guangzhou City, Guangdong Province, China. The number of infections has increased in the following 10 days and led to 5 generations of transmission. As of June 23, a total of 167 locally transmitted cases related to this outbreak were observed in 4 cities (Guangzhou, Maoming, Foshan, and Zhanjiang) in Guangdong (Figure 1A). The cases identified have been found to share the same RNA sequence as the highly infectious Delta variant strain (B.1.617.2) which was first found in India (1).

The rapid spread of the epidemic highlighted the high transmissibility of this variant strain. Based on the initial 68 infections belonging to 24 clusters that had a clear chain of transmission, we investigated the key transmission parameters including the incubation period (the period of time from infection to illness onset), the generation time (GT, the interval between infection of the primary case and secondary cases), and the serial interval (the interval between the onset of symptoms in a primary case and secondary cases). The result showed that the mean incubation period was 4.4 days $[95 \%$ confidence interval (CI): 3.9-5.0] (Figure 1B), which was shorter than that reported by $\mathrm{Li}$ et al. (4.4 vs. 5.2) in Wuhan City, Hubei Province, China (2). The mean generation time was 2.9 days (95\% CI: 2.4-3.3), which was much shorter than that reported by $\mathrm{Hu}$ et al. in Hunan Province (2.9 vs. 5.7) (2). The mean serial interval was 2.3 days (95\% CI: 1.4-3.3), which was also shorter than that reported by previous reports (2-3), and $21.6 \%(11 / 51)$ of serial intervals were negative (Figure 1C). We observed that $64.7 \%$ (44/68) of transmission events occurred during the pre-symptomatic phase, which was higher than that reported by $\mathrm{Hu}$ et al. (64.7\% vs. $59.2 \%)$ (3). The transmission parameters suggested that suppressing the rapid spread and hidden transmission of this mutant virus is of high priority.
Based on the data of the cases with illness onset (or notification) between May 18 and May 29, and the GT of 2.9 days, the basic reproductive number $\left(R_{0}\right)$ was estimated, which was defined as the expected number of additional cases that one case will generate. The estimated $R_{0}$ (maximum likelihood method) was 3.2 (95\% CI: 2.0-4.8), which was much higher than 2.2 from Li et al. (2). Based on the GT and $R_{0}$ estimated, the epidemic growth rate $(r$, which represents transmission rate of epidemic with the formula of $\left.r=\left[R_{0}-1\right] / G T\right)$ for the early stage of the outbreak was estimated as approximately 0.76 per day, which was about $100 \%$ higher than findings from previous epidemic strains (4). This result was in line with the Finlay et al. report that the transmissibility of Delta variant was increased by $97 \% \quad(95 \% \mathrm{CI}$ : 76\%-117\%) (5).

We further estimated the effective reproduction number $\left(R_{\mathrm{t}}\right)$ as of June $23(G) . R_{\mathrm{t}}$ was an indicator to measure transmission before and after the interventions (7). In this epidemic, the $R_{\mathrm{t}}$ increased from 3.0 to 3.5 from May 27 to May 29, then decreased after May 30, reached a value of 1 on June 6 , and fluctuated near 1 from June 7 to June 15. The $R_{\mathrm{t}}$ was lower than 1 from June 16 (Figure 1D), and there were no new cases reported from June 19 to June 23. To respond to the unprecedented threat, Guangdong Province has taken a series of rigorous intervention measures including mass testing, active cases finding, community management, travel restrictions, and affected area lockdown to contain this outbreak. The evidence indicated that the public health measures taken by Guangdong have had an effect on the epidemic.

In conclusion, we observed that the transmission of this COVID-19 outbreak in Guangdong was faster and more severe than that of previous epidemics. Presently, the non-pharmaceutical interventions deployed against this epidemic have been working and should be sustained in the affected area until this epidemic is fully contained. Recently, several cases 
A

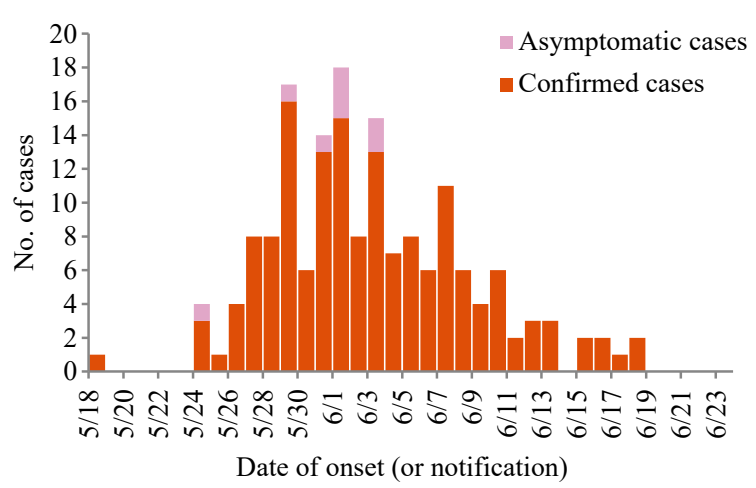

$\mathrm{C}$

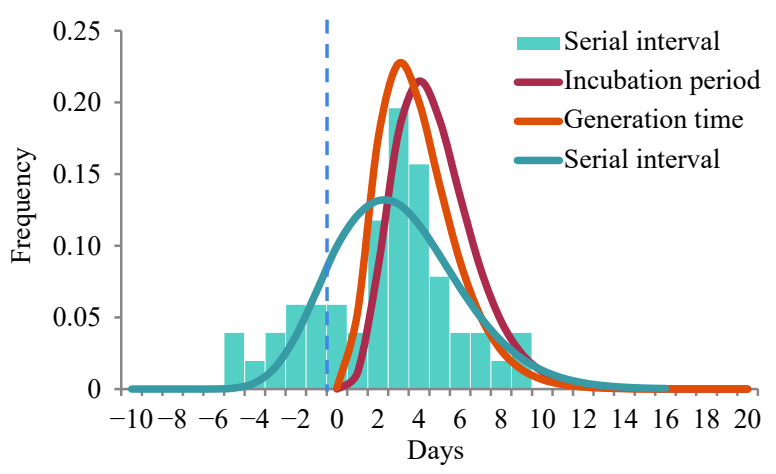

B

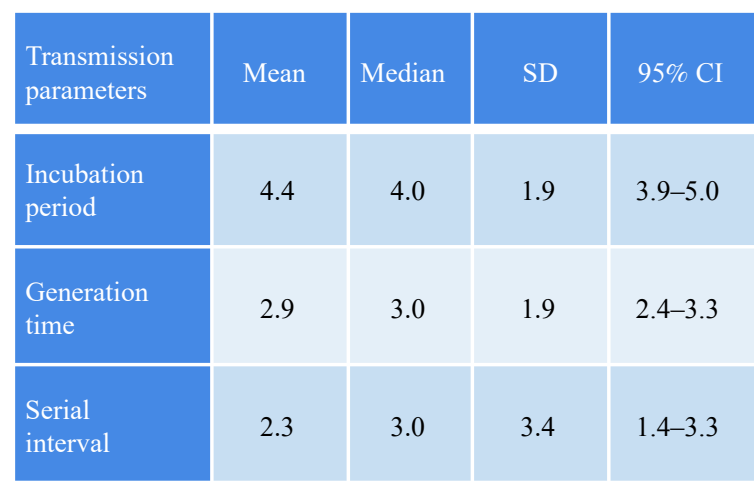

$\mathrm{D}$

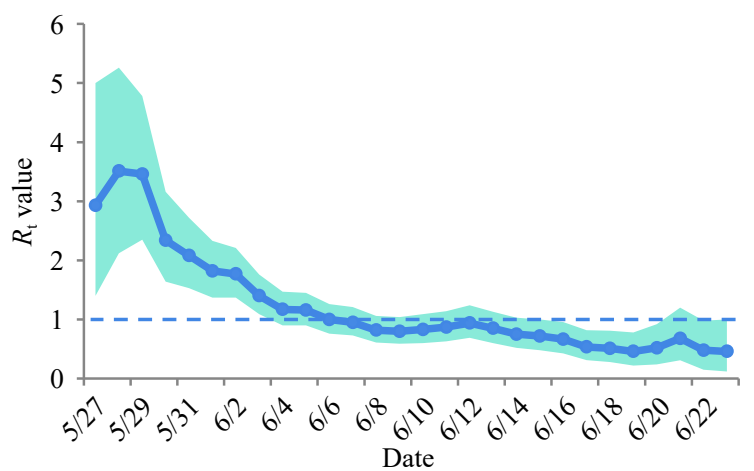

FIGURE 1. The key transmission parameters and transmission dynamics of an outbreak of the COVID-19 delta variant B.1.617.2 in Guangdong Province, China, 2021. (A) The epidemic curve of 167 COVID-19 cases in Guangdong Province China, May and June, 2021; (B) The mean, median, standard deviation (SD), and the 95\% confidence interval (Cl) of the incubation period, generation time, and serial interval; (C) The histogram of serial intervals, and the estimated distributions of the incubation period, generation time, and serial interval; (D) The effective reproduction number $\left(R_{\mathrm{t}}\right)$ of the ongoing COVID-19 epidemic in the scenarios of strict intervention measures have been implemented in Guangdong from May 27 to June 23, 2021.

Note: Distribution of the incubation period (the period of time from infection to illness onset) was estimated by log-normal distributions based on 47 confirmed cases that have a clear date of exposure and symptom onset. Distribution of the generation time (the interval between infection of the primary case and secondary cases) was estimated by gamma distributions based on 55 transmission pairs. Distribution of the serial interval (the interval between the onset of symptoms in a primary case and secondary cases) was estimated by gamma distributions based on 51 transmission pairs. In Figure 1C, the left of blue dotted line indicated serial intervals were negative, which means that the symptoms of the infectees precedes their infectors.

The daily number of reported COVID-19 cases from May 21 to June 23 and the generation time [mean: 2.9 days (SD: 1.9 days)] were used to estimate the effective reproduction number $\left(R_{\mathrm{t}}\right)$ and its $95 \%$ credible interval on each day via a 5 -day moving average using the Bayesian framework. In Figure 1D, the blue dotted line indicates $R_{\mathrm{t}}=1$, below which sustained transmission is unlikely so long as intervention measures are sustained, indicating that the outbreak is under control.

$\mathrm{R}$ software (version 3.6.0, R Core Team, Austria) was used for the data analyses, "fitdistrplus" package was used for fitting the distributions, and "EpiEstim" package was used for estimating $R_{\mathrm{t}}$.

infected by other Delta variant were observed in Shenzhen and Dongguan cities of Guangdong Province. The experience in Guangdong suggested that the emergence of variant strains would be a great threat for COVID-19 response in the scenarios of global pandemic. We should pay close attention to the prevalence of the variant strains, and nonpharmaceutical interventions should be maintained even after ongoing COVID-19 vaccination.
Funding: The Key-Area Research and Development Program of Guangdong Province (2019B111103001, 2020B111107001); The National Natural Science Foundation of China (No. 82041030).

doi: $10.46234 / \mathrm{ccdcw} 2021.148$

\# Corresponding authors: Min Kang, kangmin@yeah.net; Yan Li, lyan2009@21cn.com.

\footnotetext{
${ }^{1}$ Guangdong Provincial Center for Disease Control and Prevention, Guangzhou, Guangdong, China; ${ }^{2}$ Guangdong Provincial Institute of
} 
Public Health, Guangzhou, Guangdong, China; ${ }^{3}$ School of Public Health, Southern Medical University, Guangzhou, Guangdong, China;

${ }^{4}$ Guangzhou Center for Disease Control and Prevention, Guangzhou, Guangdong, China; ${ }^{5}$ Foshan Center for Disease Control and Prevention, Guangzhou, Guangdong, China; ${ }^{6}$ Maoming Center for Disease Control and Prevention, Guangzhou, Guangdong, China;

${ }^{7}$ Zhanjiang Center for Disease Control and Prevention, Guangzhou, Guangdong, China; ${ }^{8}$ Guangzhou Liwan District Center for Disease Control and Prevention, Guangzhou, Guangdong, China;

9 Guangzhou Panyu District Center for Disease Control and Prevention, Guangzhou, Guangdong, China; ${ }^{10}$ Guangzhou Haizhu District Center for Disease Control and Prevention, Guangzhou, Guangdong, China; ${ }^{11}$ Guangzhou Yuexiu District Center for Disease Control and Prevention, Guangzhou, Guangdong, China; ${ }^{12}$ Foshan Nanhai District Center for Disease Control and Prevention, Guangzhou, Guangdong, China; ${ }^{13}$ Maoming Dianbai District Center for Disease Control and Prevention, Guangzhou, Guangdong, China;

14 National Institute for Communicable Disease Control and Prevention, Chinese Center for Disease Control and Prevention, Beijing, China; ${ }^{15}$ Chinese Center for Disease Control and Prevention, Beijing, China.

\& Joint first authors.

Submitted: June 24, 2021; Accepted: June 25, 2021

\section{REFERENCES}

1. Adam D. What scientists know about new, fast-spreading coronavirus variants. Nature 2021;594(7861):19 - 20. http://dx.doi.org/10.1038/ d41586-021-01390-4.

2. Li Q, Guan XH, Wu P, Wang XY, Zhou L, Tong YQ, et al. Early transmission dynamics in Wuhan, China, of novel coronavirus-infected pneumonia. N Engl J Med 2020;382(13):1199 - 207. http://dx.doi.org/ 10.1056/NEJMoa2001316.

3. Hu SX, Wang W, Wang Y, Litvinova M, Luo KW, Ren LS, et al. Infectivity, susceptibility, and risk factors associated with SARS-CoV-2 transmission under intensive contact tracing in Hunan, China. Nat Commun 2021;12(1):1533. http://dx.doi.org/10.1038/s41467-02121710-6.

4. The Royal Society. Reproduction number $(R)$ and growth rate $(r)$ of the COVID-19 epidemic in the UK: methods of estimation, data sources, causes of heterogeneity, and use as a guide in policy formulation. Roy Soc 2020. https://royalsociety.org/-/media/policy/projects/set-c/set-covid-19R-estimates.pdf [2021-06-22]

5. Campbell F, Archer B, Laurenson-Schafer H, Jinnai Y, Konings F, Batra $\mathrm{N}$, et al. Increased transmissibility and global spread of SARS-CoV-2 variants of concern as at June 2021. Euro Surveill 2021;26(24) pii=2100509. http://dx.doi.org/10.2807/1560-7917.ES.2021.26.24 2100509.

6. Xiao JP, Hu JX, He GH, Liu T, Kang M, Rong ZH, et al. The timevarying transmission dynamics of COVID-19 and synchronous public health interventions in China. Int J Infect Dis 2021;103:617-23. http://dx.doi.org/10.1016/j.ijid.2020.11.005.

7. Pan A, Liu L, Wang CL, Guo H, Hao XJ, Wang Q, et al. Association of public health interventions with the epidemiology of the COVID-19 outbreak in Wuhan, China. JAMA 2020;323(19):1915-23. http://dx.doi.org/10.1001/jama.2020.6130. 